\title{
Development of a Human Factors Roadmap for the Successful Implementation of Industrial Human-Robot Collaboration
}

\author{
George Charalambous ${ }^{1,}$, Sarah Fletcher ${ }^{2}$, Philip Webb ${ }^{3}$ \\ ${ }^{1}$ SNC-Lavalin, Human Factors, Rail and Transit, London, United Kingdom \\ ${ }^{2}$ Industrial Ergonomics and Human Factors Group / \\ ${ }^{3}$ Aerostructure Assembly and Systems Installation Group Centre for Advanced Systems \\ School of Engineering, Cranfield University, United Kingdom
}

\begin{abstract}
The concept of industrial human-robot collaboration (HRC) is becoming increasingly integrated into manufacturing production lines as a means for enhancing productivity and product quality. However, developments have focused primarily on the technology and, until recently, little research has been geared to understand the key human factors (HF) that need to be considered to enable successful implementation of industrial HRC. Recent work by the authors has led to the identification of key organisational and individual level HF. The purpose of this paper is to draw together the evidence from their studies and propose a HF roadmap for the successful implementation of industrial HRC. The roadmap will have profound implications as it enables automation specialists and manufacturing system engineers to understand the key HF that need to be considered optimise the efficiency and productivity of the collaboration between humans and industrial robots.
\end{abstract}

Keywords: Human Factors Roadmap · Industrial Human-Robot Collaboration · Trust · Organisational Human Factors

\section{Introduction}

Despite the rapid integration of automated systems in manufacturing processes, a significant amount of assembly work still requires the flexibility of a human operator making the human element a vital part of the production chain [1]. In such processes, it is neither feasible nor cost-effective to introduce full automation. The manufacturing industry has shown growing interest in the concept of industrial robots working as teammates alongside human operators [2-5]. In light of recent technological developments, health and safety regulations have been updated to reflect that in some circumstances it is safe and viable for humans to work more closely with industrial robots [6]. Combining the advantages of human workers and industrial robots leads to the development of industrial human-robot collaboration (HRC). 
The International Organisation for Standardisation [6] defines HRC as a "special kind of operation between a person and a robot sharing a common workspace". Successful implementation of industrial HRC can enhance manufacturing efficiency, productivity and quality whilst reducing operating costs since the weakness of one partner can be complemented by the strengths of the other [7-10]. However, the integration of humans and robots within the same workspace can be a challenge for the human factors (HF) community. For example, the installation of large assemblies requires operators to cooperate with large and high payload robots under minimised physical safeguarding [11]. The success of such an integrated close-proximity HRC system will be determined, not only by the technical capability of the system, but also by investigating the key HF at an organisational and individual level. However, developments in the field of industrial HRC have focused primarily on the technology and, until recently, little research has been geared at understanding the organisational and individual level HF that need to be considered in order to optimise successful implementation of industrial HRC.

Recent work by the authors has led to the identification of key organisational and individual level HF $[12,13]$. Regarding the individual level HF, the authors have particularly explored the construct of trust in the robotic partner. The reason for selecting trust is because it has been widely identified in the human-robot interaction domain as a key element for the successful cooperation between humans and robots and can enhance human acceptance of robots [14-16].

The purpose of this paper is to draw together the evidence from these studies as a whole, in order to propose an initial Human Factors Roadmap for HRC implementation, which integrates all of the key factors that have been identified as important enablers to successful implementation of HRC into a set of guidance.

\section{$2 \quad$ Literature Review}

In this section we present a review of recent work carried out by the authors for the introduction of industrial HRC. Section 2.1 reviews their findings for the organisational level HF, while section 2.2 presents their work in the development of a trust scale which is specifically addressing industrial HRC.

\subsection{Organisational Level Human Factors}

Earlier literature suggests that the implementation of a technological change should not be viewed simply as an engineering problem. The impact of the change will affect the organisation and subsequently the employees. With the concept of industrial HRC still at its infancy, it is crucial to understand which organisational human factors are of most importance. To our knowledge, a framework with the key organisational human factors that need to be considered by organisations for the successful implementation of industrial HRC does not exist. Recent work by Charalambous, Fletcher and Webb [12] made the first attempt to identify the key organisational HF for the successful implementation of industrial HRC. Their work enabled to: (i) develop a theoretical framework with the key organisational human factors relevant to industrial HRC and (ii) identify whether these factors are enablers or barriers through 
an industrial exploratory case study. Although it is not the purpose of this paper to reinstate their work (detailed information can be found at [12]) a brief summary of their findings is listed below.

Major enablers: operator participation in the implementation, communication of the change to the workforce, visible senior management commitment and support to the project, provision of training to the workforce, empowerment of the workforce and existence of a process champion during the implementation.

Major barriers: lack of union involvement, lack of awareness of the manual process complexity by the system integrator, capturing the variability of the manual process prior to introducing the automated system and allocation of resources for the development of the automated system.

\subsection{Trust in industrial robots}

The development of trust is essential for the successful operation of any team [17]. Lee and See [18] defined trust as "the attitude that an agent will help achieve an individual's goals in a situation characterised by uncertainty and vulnerability" ( $\mathrm{p}$. 54). In the context of human-automation teaming, trust can influence the willingness of humans to rely on the information obtained by an automated system, particularly in risky and uncertain environments [15,19]. Lack of trust will eventually lead the operator to intervene and take control [20]. In the context of human-robot interaction (e.g. social, military and healthcare robots), earlier literature suggested that trust development can be influenced by robot attributes, such as appearance, movement, reliability and predictability. However, until recently, very little was known regarding trust development between humans and industrial robots.

Charalambous, Fletcher and Webb [13] developed a trust measurement scale suitable for industrial HRC. Although full details on the methodology can be found at [13], a summary of the scale is provided in this section. The scale identified three key components (i.e. factors) which influence human trust in industrial HRC. The factors extracted accounted for $63.5 \%$ of the total variance in the sample with a Kaiser-MayerOlkin of 0.812 while Bartlett's test of sphericity was found to be statistically significant $\left(\chi^{2}(45)=465.6, p<0.001\right)$, suggesting that the factors were unlikely to have occurred by chance. A short description of each of the key factors along with the statistic reliability achieved is provided below:

- Factor 1 - Safe co-operation: The perception held by the human operator of how safe it is to collaborate with the industrial robot. This component. This factor consisted of four items and exhibited a reliability of 0.802 .

- Factor 2 - Robot's and end-effector's reliability: The perceived reliability of the robot and the end-effector (e.g. gripping mechanism) by the human operator. This factor consisted four items and achieved a reliability of 0.712 .

- Factor 3 - Robot's motion and pick-up speed: The degree to which the robot's motion is perceive to be fluent and non-disruptive by the human operator as well as the speed at which the robot picks up and manipulates components. This factor consisted of two items and achieved a reliability of 0.612 . 


\subsection{Summary}

Taking the evidence as a whole it appears that there is an inter-relation between some of the factors at the organisational level and the developed trust scale. At the organisational level, two of the key human factors that emerged were: (i) provision of training to the workforce and (ii) operator empowerment. These two factors can be utilised along with the developed trust scale to provide a tool with which operators' trust levels in the robotic teammate can be continuously calibrated. This is described in the next section.

\section{Human Factors Roadmap for HRC Implementation: Guidance for practitioners}

The development of the roadmap is segregated in two parts, each of which provides a set of propositions:

- Part 1: It discusses how the trust scale can be utilised in an initial training programme to assist operators' initial trust calibration. The benefits of this proposition are presented. In section 3.1.

- Part 2: This part discusses how operator empowerment is vital for continuous trust calibration which in turn will dynamically optimise operators' trust in the robotic teammate. The benefits of this proposition are presented in section 3.2 .

\subsection{Part 1: Operator training programme for initial trust calibration}

To describe how training can be used to influence human operator's trust calibration in the robotic teammate, the literature from mental models will be used. When humans interact with an entity (e.g. robot), mental models are used to assist the user perceive and interpret the entity's intentions and actions [21]. At the same time, it must be noted that humans tend to have incomplete or even inaccurate mental models [22]. In an industrial HRC scenario humans will be requested to share the same workspace and collaborate with an industrial robot to complete a task. An inaccurate or incomplete mental model can potentially lead the human operator to either overestimate or underestimate the abilities of the robotic teammate. This has been described in the literature as misuse (i.e. overestimation) and disuse (i.e. underestimation) [14]. Both can be equally detrimental. The key is to achieve appropriate trust calibration. To calibrate appropriate trust in the robotic partner, it is vital for the human to hold a sufficiently developed mental model of the robot, whereby robot's capabilities are acknowledged [23]. Therefore to assist human operators to develop a sufficient mental model of their robotic teammate, it is proposed to incorporate the trust scale findings in an operator training programme.

The aim of this training programme would be to provide operators with an understanding of the robot's abilities and limitations of the key robot characteristics, rather than simply understanding how to use the robot to complete a process. This approach can help operators develop an appropriate mental model of the robot they will be requested to collaborate with. For instance, a key trust factor identified in the 
trust scale is the "perceived robot and gripping mechanism reliability". Does it mean that if the robot or the gripping mechanism is not $100 \%$ reliable all the time they are useless? According to Wickens and colleagues [24], automated systems are expected not to be perfectly reliable due to technological limitation and/or due to software and hardware failures. Therefore, in a HRC scenario it is expected that at some point, the performance of the robot (i.e. the robot itself and/or the gripping mechanism) will be less than perfect. What we need to remind ourselves is that appropriate trust calibration is primarily influenced by the "human's mental model of the robot's ability and limitations, than the ground-truth reliability of the robot itself" [23, p.63]. In other words, perception and reality are not necessarily the same and, as suggested by Merritt and Ilgen [25], trust can be heavily driven by user's perception of the robot irrespective of whether this perception is correct, partially correct or completely incorrect.

In summary, an initial training programme, before the implementation of the robotic system, could be used as a strategy to raise operators' awareness regarding the ability and limitations of the robot and assist matching operators' perceptions with the system's actual capabilities. The next section describes how operator empowerment can be used to refine human mental models of the robot and achieve continuous trust calibration.

\subsection{Part 2: Operator empowerment for continuous trust calibration}

The development of mental models is a dynamic process and these models are refined through continuous interaction [23]. Similarly, trust development is not a static process. Human trust in a system (e.g. a robot) evolves over time from dispositional (i.e. upon first encounter) to history-based trust (i.e. cumulative collaboration) [25]. As this transition occurs, humans retrieve history-based mental models to interprete the actions of the system they are working with. If the mental models created during the subsequent exposure (i.e. history-based) are not sufficiently developed, this is likely to lead to trust miscalibration. In an industrial HRC scenario, the more operators are collaborating with a robot, the more likely it is to experience a variety of real failure, errors or system deviation scenarios (particularly during the early stages of implementation). While these events occur, it is vital for operators to understand the sources of these events and the possible outcome of these events (whether a failure, error, or deviation). Also, through exposure they will be in a position to identify factors that diminish or enhance the robot's ability to perform as well as detect cues that suggest a potential malfunction. According to [22] trust can be calibrated by providing an accurate understanding of the factors that may lead the robot to fail and the outcomes of those failures. To leverage this potential and enable effective HRC, it is proposed that operator empowerment can be a key strategy.

Operator empowerment was found to be one of the key enabling organisational humans factors. In a highly complex system, higher operator control and empowerment once the system is implemented will lead to operators obtaining a better understanding of the new system and task requirement [26]. Through operator empowerment, the operators' already established mental model of the robot (from the initial training programme) will be updated based on their history of collaboration. If on the other hand, operators are not empowered but an expert is called (e.g. 
manufacturing engineer) without the operators being involved, then operators are likely to be alienated from the system. This could potentially reduce operators' ability to develop an in-depth understanding of the system's source of events (i.e. failures, errors, deviations) as well as their ability to recalibrate their trust is reduced leaving them with an incomplete mental model.

This is not to say that experts (e.g. manufacturing engineers and/or robot experts) should not be involved. Operator empowerment should not be viewed as "all or nothing". A reaction plan will be issued which will highlight the necessary steps according to the events. However, it is crucial, at all stages for the operators to be involved rather than simply turn into passive monitors of the system. This will enable them to obtain a greater understanding and awareness of the source of the event, thus making the system more transparent and understandable.

Finally, the knowledge gained by the operators, can then be passed into the training programme. Then, the training programme of future novice operators will be updated with real event scenarios. Subsequently this will accelerate appropriate trust calibration of novice operators during the initial training programme by enabling greater match between their perceptions of the system and the actual system's capabilities.

\subsection{Summary of the HRC roadmap}

The propositions suggested in sections 3.1 and 3.2 can be merged in a guiding framework for practitioners to assist appropriate operator trust calibration. The guiding framework has three key phases, each of which is described below:

Phase 1: This is shown in Figure 1. For clarity purposes, the remaining organisational human factors have not been included in the guiding framework.

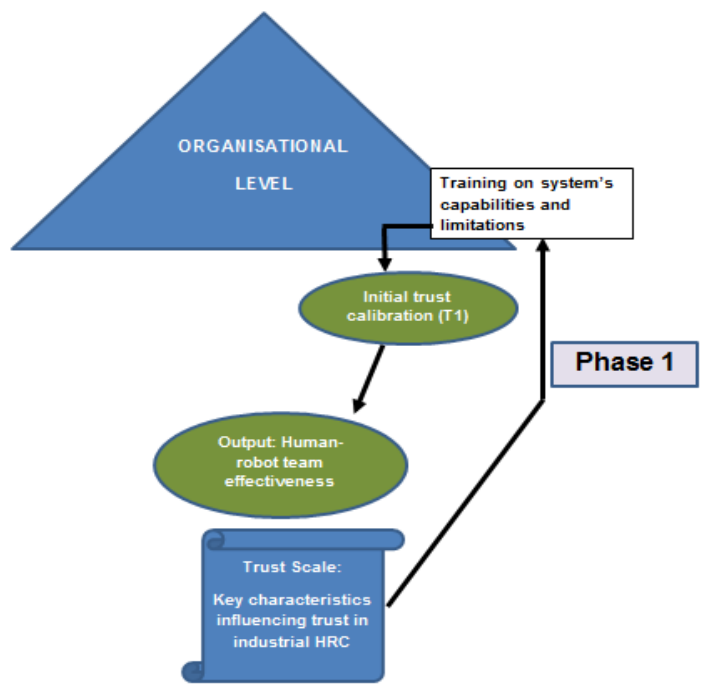

Fig. 1. Initial trust calibration via a training programme 
Phase 1 takes place when the system is still at a pre-production stage. Phase 1 suggests that the operators selected to use the robot (e.g. major users) receive training not only on how to use the robot to complete the task, but also to understand the system's capabilities and limitations as highlighted by the trust scale (i.e. perceived robot's motion; perceived robot and gripping mechanism reliability; perceived safe cooperation). This in-depth training can be provided by the system integrator (i.e. robot supplier). The training will assist operators to shape their expectations and make an initial calibration of their trust in the system (e.g. T1 on the schematic above). As operators spend more time collaborating with the robot, the experience gained during this time will start shifting their trust to history-based. Any experienced robot failures, errors or deviations will influence their mental model formation. The more they collaborate with the robot the more they will retrieve these history-based events to make sense of the robotic teammate. If their dynamic mental model formation is incomplete or inaccurate, then this will result in trust miscalibration which will eventually be reflected in the effectiveness of the team. For this reason, the second phase of the guiding framework suggests that operator empowerment is crucial.

Phase 2: Phase 2 is shown on the left hand side in Figure 2:

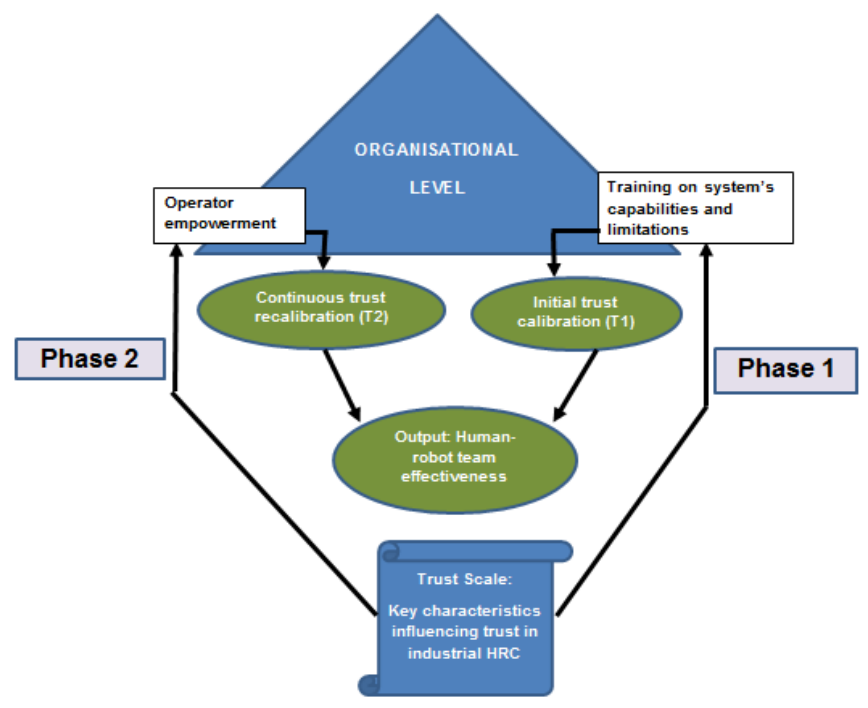

Fig. 2. Enhanced operator empowerment to enable continuous trust calibration

Empowerment will allow operators to understand the reasons behind the events, helping them to form an accurate mental model of the robot. Table 1 shows how empowerment can serve as a vehicle for or operators to achieve an accurate mental model of the robot based on historic events. 
Table 1. Dynamic trust calibration through operator empowerment.

\begin{tabular}{|c|c|c|c|c|c|}
\hline Event & $\begin{array}{l}\text { Existing operator } \\
\text { mental model is } \\
\text { challenged by the } \\
\text { event }\end{array}$ & $\begin{array}{c}\text { Operator } \\
\text { empowerment }\end{array}$ & Why did it happen? & $\begin{array}{l}\text { Operator new mental } \\
\text { model of the robot }\end{array}$ & $\begin{array}{c}\text { Impact on } \\
\text { operator's trust } \\
\text { in the robot }\end{array}$ \\
\hline $\begin{array}{l}\text { Robot } \\
\text { produces } \\
\text { an error - } \\
\text { it stops } \\
\text { operating }\end{array}$ & $\begin{array}{l}\text { "I thought the } \\
\text { robot was } \\
\text { Reliable. It } \\
\text { never did this } \\
\text { before }-I \\
\text { wonder why; is } \\
\text { something } \\
\text { wrong with it?" }\end{array}$ & $\begin{array}{c}\text { Operator } \\
\text { becomes } \\
\text { involved in } \\
\text { the } \\
\text { rectification }\end{array}$ & $\begin{array}{l}\text { E.g. Component } \\
\text { mis-positioned on } \\
\text { the fixture - } \\
\text { Therefore, the opera- } \\
\text { tor now can } \\
\text { understand how } \\
\text { the robot "reads" } \\
\text { the position of the } \\
\text { component }\end{array}$ & $\begin{array}{l}\text { "This robot is very } \\
\text { sensitive to material } \\
\text { positioning - I must } \\
\text { inspect more } \\
\text { carefully the } \\
\text { positioning of the } \\
\text { component on the } \\
\text { fixture" }\end{array}$ & $\begin{array}{c}\text { Trust is } \\
\text { recalibrated } \\
\text { based on } \\
\text { this event. }\end{array}$ \\
\hline
\end{tabular}

Assume the robot produces an error and stops operating (first column of the table). This anomaly, challenges operator's existing mental model of the robot operating reliably (second column of the table). The operator is empowered to take rectification action and/or be part of the recovery process (third column of the table). This assists the operator to understand the source of the error as well as understand how the robot's system operates (fourth column of the table - how it "reads" the position of the component). This new knowledge assists the operator to mould a new mental model based on this event (fifth column of the table). Subsequently, his or her trust in the robotic teammate is recalibrated. If for example, the "Action" (third column) did not take place, then the operator would not be in a position to understand the reason for the error, hence leaving them with an outdated mental model. Subsequently, the operator will attempt to update their outdated mental model based on their perception, potentially leading to trust miscalibration.

Phase 3: Finally, in phase 3, the knowledge gained by the exposure is fed into the training programme which will then be used to accelerate appropriate trust calibration for future novice operators. This is shown by the "Phase 3" arrow in Figure 3 which completes the guiding framework: 


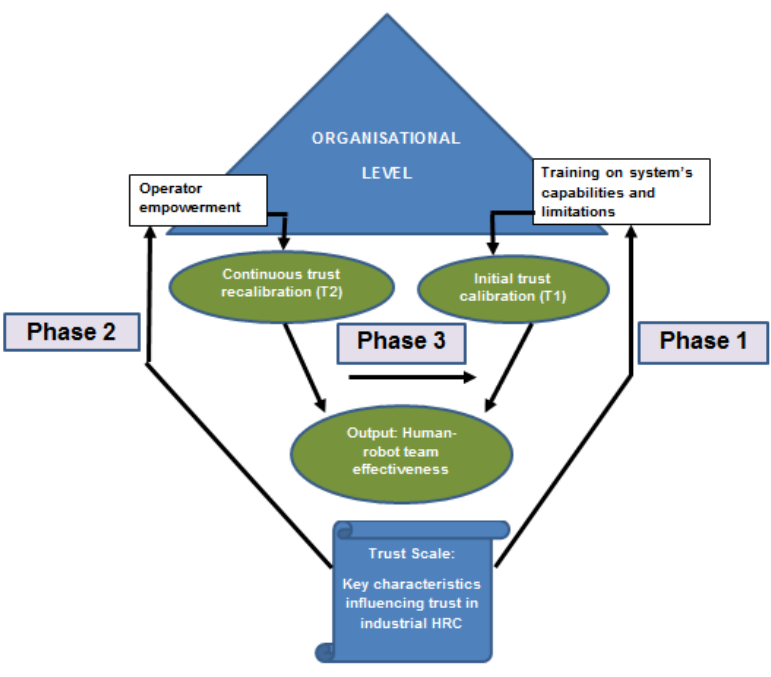

Fig. 3. The finalised guiding framework for calibrating appropriate levels of operators' trust

\section{Conclusion}

Until recently, very little work was geared to understand the human factors for the successful implementation of industrial HRC. Recent work by the authors has identified a number of organisational HF. Also, the authors explored the construct of trust in the robotic partner. This work led to the development of a scale to evaluate trust in industrial HRC.

This paper draws together the evidence from these studies as a whole and proposes an initial HF roadmap for the successful implementation of industrial HRC. The roadmap provides propositions in a guiding framework for practitioners to assist appropriate trust calibration to the robotic teammate:

- Training programme: First, it is proposed that a training programme is developed which will incorporate the robot's key characteristics identified in the trust scale (i.e. perceived safety, perceived reliability and robot motion and pick-up speed). The training programme will enable operators understand the abilities as well as limitations of the robotic teammate, rather than simply receiving training on how to use the robot to complete a process. By openly addressing the actual capabilities of the robot, will enable human operators to develop an appropriate, and more realistic, mental model of the robot they will be requested to collaborate with.

- Operator empowerment: As operators gain additional experience collaborating with the robotic teammate, it is crucial to enhance operator empowerment particularly during degraded events, such as robot failures, errors or deviations. Empowering operators (along with robot specialists) will allow 
them to understand the reasons behind the events, helping them to form an accurate mental model of the robot.

By employing this approach, automation specialists and manufacturing system designers can dynamically calibrate human workers' trust in the robotic partner to optimise the efficiency and productivity of the collaboration.

\section{References}

1. Ding Z, Hon B.: Constraints analysis and evaluation of manual assembly. CIRP AnnalsManufacturing Technology, 62(1):1-4 (2013).

2. Hägele M, Schaaf W, Helms E.: Robot assistants at manual workplaces: Effective cooperation and safety aspects. International symposium on robotics ISR 2002 / CD-ROM. October 7-11, Stockholm, (2002).

3. Schraft RD, Meyer C, Parlitz C, Helms E.: Powermate - a safe and intuitive robot assistant for handling and assembly tasks. IEEE international conference on robotics and automation, IEEE, Barcelona, pp 4047-4079, (2005).

4. Santis AD, Siciliano B, Luca AD, Bicchi A.: Atlas of physical human-robot interaction. Mech Mach Theor 43(3):253-270, (2008).

5. Unhelkar VV, Siu HC, Shah JA.: Comparative performance on human and mobile robotic assistants in collaborative fetch-and deliver tasks. Human Robot interaction 2014. ACM, Bielefeld, (2014).

6. International Organisation for Standardisation: Robots and robotic devices-safety requirements for industrial robots, Part 1: robots. international standards organisation, Geneva (2011).

7. Bortot D, Born M, Bengler K.: Directly or detours? How should industrial robots approach humans?. ACM/IEEE international conference on human-robot interaction (HRI), IEEE, (2013)

8. Papadopoulos AV, Bascetta L, Ferretti G.: Generation of Human Walking Paths. International Conference on Intelligent Robots and Systems (IROS), Tokyo, Japan, (2013).

9. Weidner R, Kong N, Wulfsberg JP.: Human Hybrid Robot: a new concept for supporting manual assembly tasks. Production Engineering Research Development, 7(6):675-684, (2013).

10.Zhang WJ, Van Luttervelt CA.: Toward a resilient manufacturing system. CIRP Annals Manufacturing Technology, 60(1): 469-472, (2011)

11.Walton M, Webb P, Poad M.: Applying a concept for robot-human cooperation to aerospace equipping processes, SAE International, (2011).

12.Charalambous G, Fletcher S, Webb P.: Identifying the key organisational human factors for introducing human-robot collaboration in industry: an exploratory study. The International Journal of Advanced Manufacturing Technology 81 (9-12): 2143-2155, (2015).

13.Charalambous, G., Fletcher, S., Webb, P.: The Development of a Scale to Evaluate Trust in Industrial Human-robot Collaboration. International Journal of Social Robotics, 1-17, (2015).

14.Parasuraman R, Riley V.: Humans and automation: use, misuse, disuse, abuse. Hum Fact 39(2):230-253, (1997).

15.Freedy A, de Visser E, Weltman G, Coeyman N.: Measurement of trust in human-robot collaboration. In: Proceedings of the 2007 international conference on collaborative technologies and systems, Orlando (2007).

16.Chen JY, Barnes MJ.: Human-agent teaming for multirobot control: a review of human factors issues. IEEE Trans Hum Mach Syst 44(1):13-29 IEEE, (2014). 
17.Groom V, Nass C.: Can robots be teammates? Benchmarks in human-robot teams. Interact Stud 8(3):483-500, (2007).

18.Lee JD, See KA.: Trust in automation: designing for appropriate reliance. Hum Fact 46(1):50-80, (2004).

19.Park E, Jenkins Q, Jiang X.: Measuring trust of human operators in new generation rescue robots. Proceedings of the 7 th JFPS international symposium on fluid power, Toyom, pp 15-18, (2008).

20.de Visser EJ, Parasuraman R, Freedy A, Freedy E, Weltman G.: A comprehensive methodology for assessing human-robot team Performance for use in training and simulation. Proceedings of the 50th Human factors ergonomics society, San Francisco, pp. 2639-2643, (2006).

21.Phillips, E., Ososky, S., Grove, J., Jentsch, F.: From tools to teammates: Toward the development of appropriate mental models for intelligent robots. Proceedings of the 55th Annual Meeting of the Human Factors and Ergonomics Society, (pp. 1491-1495), (2011)

22.Ososky, S., Sanders, T., Jentsch, F., Hancock, P., Chen, J. Y.: Determinants of system transparency and its influence on trust in and reliance on unmanned robotic systems. Proceedings of SPIE, Unmanned Systems Technology XVI, 90840E, (pp. 1-12), (2014).

23.Ososky, S., Schuster, D., Phillips, E., Jentsch, F.: Building appropriate trust in human-robot teams. Trust and Autonomous Systems AAAI Spring Symposium (pp. 60-65). AAAI, (2013).

24.Wickens, C. D., Huiyang, L., Santamaria, A., Sebok, A., \& Sarter, N.: Stages and levels of automation: An integrated meta-analysis. In the Proceedings of the 54th Annual Human Factors and Ergonomics Society Meeting, (pp. 383-393), (2010).

25. Merritt, S. M., \& Ilgen, D. R.: Not all trust is created equal: Dispositional and history-based trust in human-automation interactions. Human Factors: The Journal of the Human Factors and Ergonomics Society, 50(2), 194-210, (2008).

26.Wall, T. D., Cordery, J. L., Clegg, C. W.: Empowerment, performance, and operational uncertainty: A theoretical integration. Applied Psychology: An International Review, 51(1), 146-169, (2002). 


\title{
Development of a human factors
}

roadmap for the successful

implementation of industrial

human-robot collaboration

\author{
Fletcher, Sarah R.
}

Springer

George Charalambous, Sarah Fletcher and Plilip Webb, (2016) Development of a human factors roadmap for the successful implementation of industrial human-robot collaboration, Advances in Ergonomics of Manufacturing: Managing the Enterprise of the Future: Proceedings of the AHFE 2016 International Conference on Human Aspects of Advanced Manufacturing, July 27-31, 2016, Walt Disney World, Florida, USA, pp195-206

http://dx.doi.org/10.1007/978-3-319-41697-7_18

Downloaded from Cranfield Library Services E-Repository 